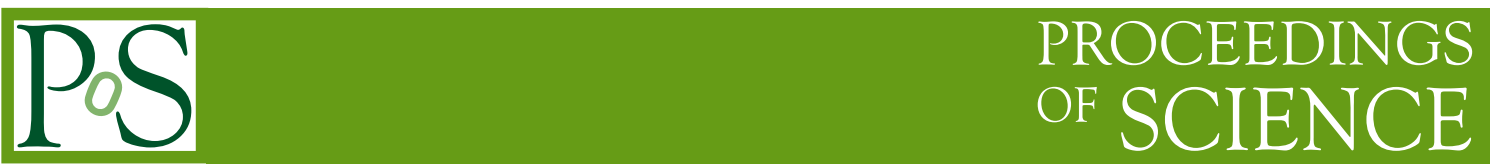

\title{
Status of the DUNE experiment
}

\author{
J. V. Dawson, for the DUNE collaboration* \\ APC, AstroParticule et Cosmologie, Université Paris Diderot, CNRS/IN2P3, CEA/lrfu, \\ Observatoire de Paris, Sorbonne Paris Cité, 10, rue Alice Domon et Léonie Duquet, 75205 Paris \\ Cedex 13, France \\ E-mail: jdawson@in2p3.fr
}

The Deep Underground Neutrino Experiment (DUNE) aims to make precise measurements of long-baseline neutrino oscillations over a $1300 \mathrm{~km}$ baseline. The DUNE Far Detectors will be 10 kton Liquid Argon TPCs which require a dedicated prototyping effort (ProtoDUNE), currently ongoing at CERN. With gigantic Far Detectors, deep underground, DUNE will be able to detect neutrinos from a Galactic core-collapse supernova, should one occur, and search for nucleon decay and other physics beyond the Standard Model.

European Physical Society Conference on High Energy Physics - EPS-HEP2019 -

10-17 July, 2019

Ghent, Belgium

* Speaker. 


\section{Introduction}

The Deep Underground Neutrino Experiment (DUNE) is a next generation neutrino oscillation experiment [1,2]. A high power wide-band beam operating in neutrino (anti-neutrino) mode will be produced at Fermilab, the flux and flavour composition will be characterised with the Near Detector. At a baseline of 1,300 km, deep underground at the Sandford Underground Research Facility (SURF, South Dakota), four gigantic Far Detector modules will measure $v_{\mu}\left(\bar{v}_{\mu}\right)$ disappearance, $v_{e}\left(\bar{v}_{e}\right)$ and $v_{\tau}\left(\bar{v}_{\tau}\right)$ appearance with the goals of:

- determining the Neutrino Mass Ordering (at more than 5 sigmas)

- measure the CP Violating phase over a wide range of values

- measuring precisely the oscillation parameters

- testing the 3-flavour paradigm

The experiment is conceived to be highly sensitive to the matter effect with a long baseline of 1300 $\mathrm{km}$. At this distance, DUNE will be able to unambiguously determine both the Mass Hierarchy and CP Violating phase $\delta_{C P}$. Thanks to the wide-band high intensity neutrino (anti-neutrino) beam from Fermilab, planned to start in 2026, DUNE will make a spectral measurement, covering both the first and second oscillation maxima as shown in Figure 1. Both the neutrino mass ordering and CP Violating phase affect differently the amplitudes and positions of the two maxima, giving additional power to the measurement.
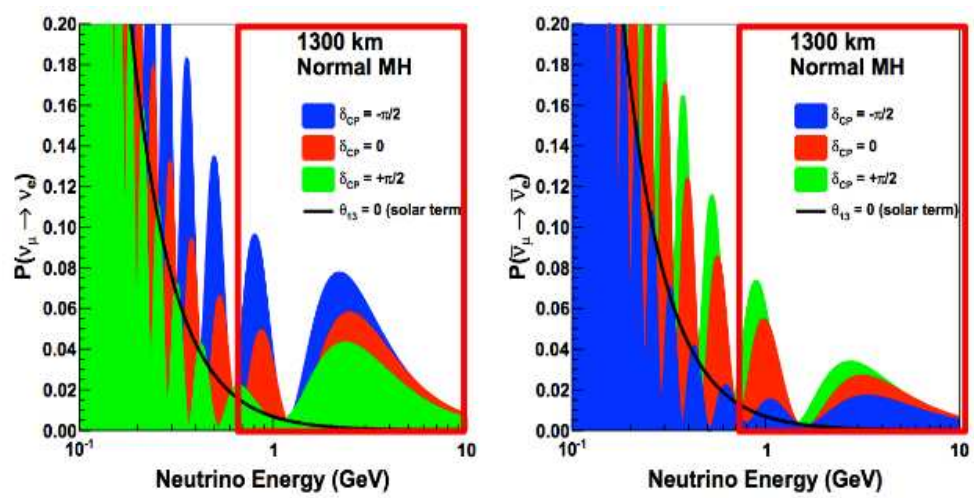

Figure 1: Left, the oscillation probability $v_{\mu} \rightarrow v_{e}$ at the DUNE baseline of $1300 \mathrm{~km}$ [2]. Right, the antineutrino equivalent. The red box denotes the energy range of interest for DUNE.

DUNE requires a high power wide-band beam which is briefly discussed in Section 2.1, Near Detectors to fully characterise the beam and extrapolate the unoscillated spectrum at the Far Detector see Section 2.2. The DUNE Far Detector modules are discussed in Section 2.3, they will be Liquid Argon TPCs (LArTPC), each holding a fiducial mass of 10 ktons [3]. These TPCs are the subject of extensive prototyping as discussed in Section 2.4. With huge detectors deep underground, DUNE has large subsidary science program which includes the potential observation of a Galactic Core Collapse Supernova, see Section 3.1 and the search for Nucleon Decay, see Section 3.2 . 


\section{The DUNE Experimental Setup}

\subsection{Neutrino beam}

The DUNE neutrino beam will be an intense wide-band beam produced by protons from Fermilab's main injector with energies of $60-120 \mathrm{GeV}$. The spectrum has been optimised to enhance sensitivity to the measurement of the CP Violating phase. The beam is due to begin operation at a power of 1.2 MW in 2026, which will be upgradable to $2.4 \mathrm{MW}$ by 2032. The DUNE beam will operate in both neutrino and anti-neutrino modes.

\subsection{Near Detector System}

The Near Detectors lie $575 \mathrm{~m}$ downstream of the beam, their primary goal is to predict the neutrino spectrum at the Far Detector, which can be broken down into the following objectives:

- Measure interactions on Argon

- Measure the neutrino energy

- Constrain the cross-section model

- Measure the neutrino flux

- Obtain data with different fluxes

- Monitor the neutrino beam

Three different detectors are conceived to do this; a Liquid Argon TPC (LArTPC), a MultiPurpose Detector (MPD) and a Scintillating Tracking Spectrometer (3DST-S).

The LArTPC is a liquid Argon TPC based on a similar technology to the Far Detector. The baseline design LArTPC is $7 \mathrm{~m}$ wide $\mathrm{x} 3 \mathrm{~m}$ high $\mathrm{x} 5 \mathrm{~m}$ deep. Its fiducial mass will be large enough to ensure the capability to measure $v_{e}$-electron scattering, profitting from the well-known crosssection to make an absolute flux normalisation of the beam. However, a significant proportion of muons produced by neutrino interactions in the LArTPC will escape the detector. To account for this the MPD sits behind the LArTPC.

The MPD is a magnetized High Pressure gaseous Argon-Methane (10 bar 90-10 $\mathrm{Ar}^{-} \mathrm{CH}_{4}$ mixture) TPC with calorimeter (ECAL). The gaseous TPC with magnetic field will be able to measure with high resolution the momentum of these forward-going muons which escaped the LArTPC. The MPD will also be able to measure $v$-Ar interactions with low threshold and acheive exquisite Particle IDentification, separating neutrino and anti-neutrino interactions.

Both the LArTPC and MPD are movable, allowing measurements of a variety of different off-axis angles and hence mean beam neutrino energies. This is the DUNE-PRISM concept.

The 3D Scintillating Tracking Spectrometer (3DST-S) remains fixed in place, on axis, as a neutrino beam monitor. This detector comprises a matrix of $1 \mathrm{~cm}^{3}$ scintillator cubes read-out with orthogonal optical fibers in three dimensions. This development is the same as T2K ND280 upgraded called 'Super-FGD'. The tracker will have excellent $4 \pi$ acceptance and fast timing. The fast timing will allow tagging of neutrons from recoils and determining their energy from time-offlight. This detector also provides a Carbon target for neutrino interactions 


\subsection{Far Detectors}

Liquid Argon TPCs provide a means to constructing gigantic detectors ( $\sim 60 \mathrm{~m}$ long, $12 \mathrm{~m}$ high and $12 \mathrm{~m}$ wide) with excellent calorimetric and spatial resolving power. The DUNE TPCs are conceived to make fine-grained ( $\sim 3 \mathrm{~mm}$ pitch) 'images' of the ionization tracks from the products of neutrino interactions in the liquid argon. Two different technologies are proposed for the readout of the drifted ionization charge, which can be measured either by induction and/or collected directly in the liquid (Single Phase detector) or after extraction and multiplication in a layer of gaseous argon (Dual Phase detector).

These two charge readout strategies result in very different designs for a DUNE 10 kton module. For Single-Phase, in which charge signals are formed from three wire planes; two induction and one collection. Here the drift length of the detector is limited to 3 to 4 metres, such that a DUNE module comprises several TPCs within the liquid argon volume [4]. The Dual-Phase is a monolithic design, the signal-to-noise achievable with its two collection anodes allows for a $12 \mathrm{~m}$ drift length [5].

\subsection{The ProtoDUNEs}

The ProtoDUNEs, one Single-Phase and one Dual-Phase, are a necessary R\&D step towards the construction of the DUNE Far Detectors. With them all the engineering solutions and installation procedures will be tested. Full-size components identical to those proposed for the Far Detector will be used. The prototypes are the largest liquid argon TPCs ever constructed, holding several hundreds of tonnes of liquid argon. With these large detectors, the long term performance and stability will be demonstrated.

Construction of the Single Phase ProtoDUNE [6] was completed during 2018, and it successfully took data with a charged particle beam. This test beam data will allow characterisation of the detector response over the energy range of interest for DUNE ( $\sim 0.5$ to $8 \mathrm{GeV})$. ProtoDUNE Dual-Phase [7], was filled with liquid argon during August 2019 and the first cosmic ray muon tracks have been seen. It will be characterised first using cosmic ray muons.

\section{The DUNE Physics Program}

The DUNE oscillation physics program includes the measurement of the $\delta_{c p}$ phase, deterimination of the neutrino mass ordering, measurement of the mixing angle $\theta_{23}$ and the determination of its octant, and sensitive tests of the three-neutrino paradigm.

The most recent sensitivity study, due to be published in the DUNE Technical Design Report, uses more realistic simulations and reconstruction methods than in previous studies. The detector simulations are based on LArSoft and use the GENIE event generator, GEANT4 particle propogation and a realistic detector read-out simulation. To determine the neutrino energy, the charged lepton and hadronic shower energies are reconstructed separately and then summed. When the charge lepton is a muon, two methods to obtain the lepton energy are employed. If the muon is contained, the range is used, whereas if the muon escapes the detector, the Multiple Coulomb Scattering method is used. The identification of the neutrino flavour is met by identifying individual particles. This neutrino flavour identification is accomplished using a modern convolutional neural 
network technique. The selection efficiency obtained, in this way, is slightly higher than that used in the DUNE Conceptual Design Report (CDR) [1]. The expected sensitivity to the neutrino mass ordering for all possible values of the $\mathrm{CP}$ Violating phase $\left(\delta_{c p}\right)$ as greater than $5 \sigma$ after 7 years of data taking. The expected sensitivity for the CP Violating phase is greater than $3 \sigma$ for $75 \%$ of $\delta_{c p}$ values after 10 years. Figure 2 shows the sensitivities as a function of the $\mathrm{CP}$ violating phase.
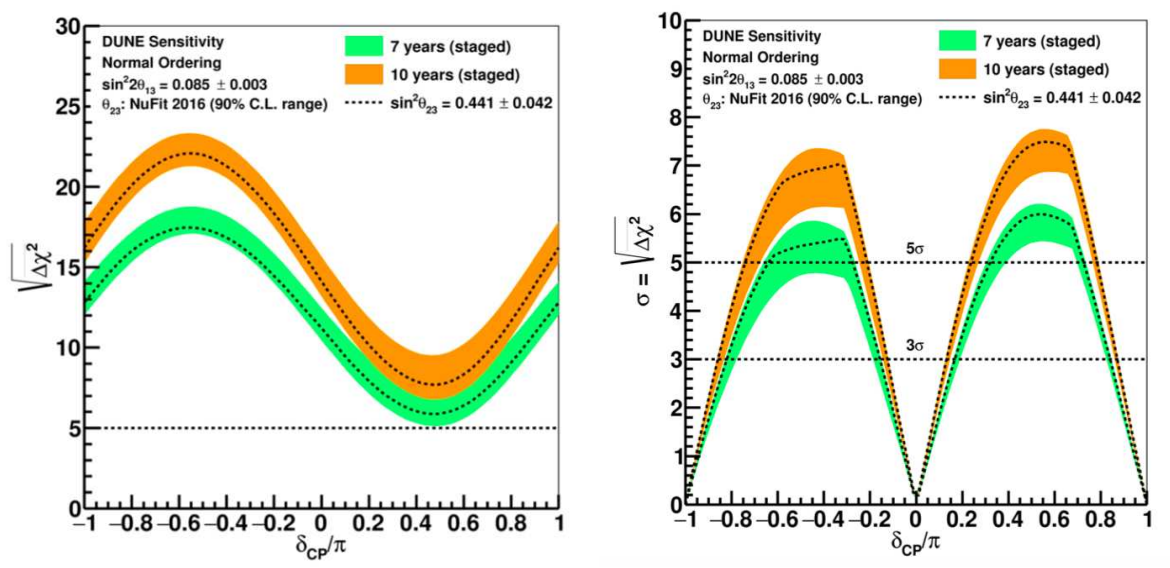

Figure 2: Sensitivity of DUNE to the neutrino mass ordering (left) and CP violating phase (right). Green and yellow bands denote the staging assumptions, 7 years (exposure of $300 \mathrm{kt}$ MW years) and 10 years (exposure of $556 \mathrm{kt} \mathrm{MW}$ years) respectively. The width of the bands indicates variation in possible central values of $\theta_{23}$.

\subsection{SuperNova Burst}

One of the primary science goals of DUNE is to detect and measure neutrinos from a corecollapse supernova within our galaxy, should one occur. Other massive neutrino detectors worldwide are based on water or organic liquid scintillators and are therefore dominantly sensitive to $\bar{v}_{e}$ detected via the Inverse Beta Decay process on hydrogen. DUNE, however, uses liquid argon as its neutrino target and is therefore uniquely sensitive to $v_{e}$ via the reaction $v_{e}+{ }^{40} \mathrm{Ar} \rightarrow e^{-}+{ }^{40} K^{*}$. In this reaction the observable is the emitted electron and de-excitation products from the excited ${ }^{40} K^{*}$ state. For a galactic core collapse supernova at $10 \mathrm{kpc}$, some 1000 neutrino interactions of this type are expected in the Far Detector. This burst is expected to last $\sim 10$ seconds with typical energies between 5 and $30 \mathrm{MeV}$.

DUNE will be dominantly sensitive to $v_{e}$, however, all flavour Neutral Current scattering $v_{x}+$ $A r \rightarrow v_{x}+A r^{*}$, which results in a gamma ray cascade from the excited state Ar nucleus, is also being studied.

\subsection{Beyond the Standard Model Physics}

DUNE has a wide non-beam science program which includes the search for Beyond the Standard Model Physics.

The search for Nucleon Decay is another primary physics goal of DUNE. DUNE is sensitive to $p \rightarrow K^{+} v$ which is expected to have a lifetime greater than $10^{33}$ years in SUSY models. Other decay modes channels, such as $n \rightarrow K^{+} e^{-}, p \rightarrow l^{+} K^{o}$ and $p \rightarrow \pi^{o} e^{+}$are also being studied. 
Another baryon number violating process is neutron-antineutron oscillations, which would produce a characteristic 'star' signature, made up of charged and neutral pions, in the Far Detector.

Other BSM physics topics include the search for new particles: light dark matter, boosted dark matter and heavy neutral leptons. DUNE will search for deviations from the PMNS neutrino mixing paradigm, exploring the possibility of non-standard neutrino interactions, non-unitarity of the PMNS matrix, CPT or Lorentz violation, and large extra dimensions etc.

\section{Conclusion}

DUNE will measure neutrino and anti-neutrino oscillations over a $1300 \mathrm{~km}$ baseline with 4 $\times 10 \mathrm{kt}$ fiducial mass LArTPCs. Large underground detectors will provide other opportunities for interesting physics studies, in particular the search for Nucleon decay, neutron-anti-neutron oscillations, and the potential to observe neutrinos from a Galactic SuperNova etc.

The LArTPCs technology development is well underway with protoDUNEs at CERN. Both Single-Phase and Dual-Phase protoDUNEs are operational. The Single Phase began running in 2018, and it successfully took data with a charged particle beam.

The DUNE Technical Design Report for both Single-Phase and Dual-Phase detector designs will be public shortly. The first DUNE detector is due to be ready by 2024, with data-taking with beam from 2026.

\section{References}

[1] DUNE collaboration, Long-Baseline Neutrino Facility (LBNF) and Deep Underground Neutrino Experiment (DUNE): Conceptual Design Report, Volume 1: The LBNF and DUNE Projects, 1601.05471.

[2] DUNE collaboration, Long-Baseline Neutrino Facility (LBNF) and Deep Underground Neutrino Experiment (DUNE): Conceptual Design Report, Volume 2: The Physics Program for Dune at LBNF, 1512.06148.

[3] R. Acciarri, M. A. Acero, M. Adamowski, C. Adams, P. Adamson, S. Adhikari et al., Long-Baseline Neutrino Facility (LBNF) and Deep Underground Neutrino Experiment (DUNE) Conceptual Design Report, Volume 4 The DUNE Detectors at LBNF, ArXiv e-prints (2016) [1601.02984].

[4] DUNE collaboration, The DUNE Far Detector Interim Design Report, Volume 2: Single-Phase Module, 1807.10327.

[5] DUNE collaboration, The DUNE Far Detector Interim Design Report, Volume 3: Dual-Phase Module, 1807.10340 .

[6] DUNE collaboration, The Single-Phase ProtoDUNE Technical Design Report, 1706.07081.

[7] I. De Bonis et al., LBNO-DEMO: Large-scale neutrino detector demonstrators for phased performance assessment in view of a long-baseline oscillation experiment, 1409.4405. 